\title{
AVALIAÇÃO DO DESEMPENHO DOS PAÍSES NOS JOGOS PAN-AMERICANOS E VERIFICAÇÃO DA OCORRÊNCIA DE HOME ADVANTAGE
}

\author{
Fábio Gomes Lacerda ${ }^{1}$, Maria Cecília de Carvalho Chaves $^{2}$, \\ Silvio Figueiredo Gomes Júnior ${ }^{3}$, João Carlos C.B. Soares de Mello ${ }^{*}$ \\ e Eliane Ribeiro Pereira ${ }^{5}$
}

Recebido em 19 de dezembro 2008 / Aceito em 15 de fevereiro 2011

\begin{abstract}
In recent years, many academic papers have been developed to verify the advantage in sports competitions play in their own fields (Home Advantage). Many of these works use statistical tools to assess the occurrence of Home Advantage and its causes. This article proposes the application of a multicriteria methodology to assess the occurrence of Home Advantage in the Pan American Games, analyzing the advantages and disadvantages of this method compared to commonly used. The work focuses in particular the Dominican Republic and Argentina delegations performance along the Pan American Games history. The results indicate the advantage of the countries analyzed in hosting competitions.
\end{abstract}

Keywords: Home Advantage, Pan American Games, multicriteria methodology.

RESUMO. Nos últimos anos, muitos trabalhos acadêmicos vêm sendo desenvolvidos para se verificar a existência de vantagem em disputar competições esportivas em domínios próprios (Home Advantage). Um grande número destes trabalhos utiliza ferramentas estatísticas para verificar a ocorrência do Home Advantage e suas causas. Este artigo propõe a aplicação de uma metodologia multicritério para verificar a ocorrência de Home Advantage nos Jogos Pan Americanos. O trabalho foca em especial o desempenho da delegação da República Dominicana e da Argentina ao longo da história dos jogos Pan Americanos, analisando as vantagens e desvantagens de utilização deste método em relação aos usualmente utilizados. Os resultados indicam a vantagem dos países analisados em sediar as competições.

Palavras-chave: Home Advantage, Jogos Pan-Americanos, método multicritério.

\footnotetext{
*Autor correspondente - E-mail: joaocsmello@gmail.com

$1_{1}$ Mestrado em Engenharia de Produção, Universidade Federal Fluminense.

2 Departamento de Engenharia Industrial, Pontifícia Universidade Católica do Rio de Janeiro.

${ }^{3}$ Doutorado em Engenharia de Produção, Universidade Federal Fluminense.

${ }^{4}$ Departamento de Engenharia de Produção, Universidade Federal Fluminense.

${ }^{5}$ Departamento de Ciências Contábeis, Universidade Federal do Rio de Janeiro.
} 


\section{INTRODUÇÃO}

Sistemas de avaliação em esportes têm sido objeto de estudo de diversos autores que buscam desenvolver uma forma "justa" de ordenação dos competidores. Os primeiros trabalhos neste sentido buscavam a elaboração de índices com incorporação de indicadores sócio-econômicos, como, por exemplo, PIB, tamanho da população, etc. Lozanno et al. (2002) e Churilov \& Flitman (2006) utilizaram a metodologia Análise Envoltória de Dados (DEA - Data Envelopment Analysis) para elaborar um ranking dos Jogos Olímpicos. Lins et al. (2003) fizeram uso de uma variação do método DEA, chamado ganhos de soma zero, para analisar os resultados das olimpíadas de Sidney em 2000; Soares de Mello et al. (2008) propuseram um método que contemplava os jogos de inverno de Salt Lake City, englobados nas Olimpíadas de Sydney, em 2000, que utilizava DEA com restrição aos pesos e avaliação cruzada.

Independentemente da forma utilizada para a ordenação dos competidores, é senso comum que o objetivo de qualquer competidor é a vitória. Diversos são os fatores que podem determinar a vitória de uma equipe em uma competição esportiva. Notadamente, em esportes de alto rendimento como as modalidades olímpicas, as equipes se preparam cada vez mais para as competições, utilizando-se de recursos até então não aplicados, como pesquisas científicas e análises de desempenho dos adversários baseados em ferramentas estatísticas.

Dentre os fatores que mais influenciam o desempenho das equipes, o local onde a competição ocorre tem sido apontado como um dos mais determinantes. A vantagem de uma equipe mandante em jogar nos seus domínios, Home Advantage, vem sendo quantificada considerando-se diferentes enfoques, tanto subjetivos quanto objetivos. Um exemplo de enfoque objetivo na análise de Home Advantage é o simples levantamento de pontos conquistados em uma competição disputando jogos em casa e como visitante. A diferença de desempenho apontada pode indicar para um treinador ou dirigente qual é a melhor estratégia de preparação que a equipe deve desenvolver. Um enfoque subjetivo para análise de Home Advantage é a verificação do impacto da presença de torcedores na motivação dos jogadores, ou ainda a influência exercida pelo público no julgamento dos árbitros durante as partidas (Balmer et al., 2001, 2003; Nevill et al., 2002). A análise de Home Advantage é um dos temas que mais tem rendido trabalhos estatísticos e de Pesquisa Operacional aplicada aos esportes, indicando um terreno fértil para o desenvolvimento de trabalhos científicos que visem o aumento de rendimento e a construção de estratégias de preparação inovadoras para as equipes.

Alguns métodos de ordenação e análise de desempenho de equipes foram desenvolvidos. O método lexicográfico é o mais utilizado, como mostrado em Lins et al. (2003). Sua principal desvantagem, no caso de Jogos Olímpicos ou Jogos Pan Americanos, é que uma única medalha de ouro tem maior valor do que qualquer número de medalhas de prata, valendo o mesmo raciocínio para estas com relação às de bronze. A valorização excessiva das medalhas de ouro, em detrimento das de prata ou bronze pode levar uma delegação que teve um bom desempenho em várias modalidades a ter uma classificação pior do que outra que tenha tido um desempenho pior no conjunto de modalidades, mas um número maior de primeiros lugares.

Desta limitação do método lexicográfico surgiram os vários métodos de ordenamento, normalmente baseados em DEA (Lozano et al., 2002; Lins et al., 2003; Churilov \& Flitman, 2006; Li 
et al., 2008; Soares de Mello et al., 2008, 2009). Neste trabalho é usado um método alternativo para verificar a existência de Home Advantage.

Este artigo está estruturado da seguinte forma: inicialmente é realizada uma breve revisão bibliográfica dos estudos mais recentes sobre Home Advantage, enfatizando os métodos utilizados e seus objetivos. A seguir, é sugerido um modelo multicritério para gerar uma nova ordenação entre os competidores. Finalmente, a metodologia é aplicada para verificação de ocorrência de Home Advantage, sendo utilizadas como exemplo as participações da República Dominicana e da Argentina nos jogos Pan Americanos.

\section{ESTUDOS DE HOME ADVANTAGE E SISTEMAS DE AVALIAÇÃO EM ESPORTES}

A maioria dos artigos acadêmicos utiliza-se de técnicas de estatística e testes paramétricos para quantificar o desempenho de equipes dentro e fora de seus domínios. Balmer et al. (2001) realizam uma avaliação de Home Advantage entre os países participantes dos Jogos Olímpicos de Inverno, baseando-se em testes de hipóteses estatísticas para enfoques objetivos e subjetivos, atribuindo pesos diferentes para medalhas de Ouro, Prata e Bronze, através de pontuações arbitradas. Posteriormente, os mesmos autores (Balmer et al., 2003) repetiram a análise para os Jogos Olímpicos de Verão, testando sua técnica para um conjunto de dados maior que o anterior. Pollard (2002) analisa a redução da vantagem de uma equipe em jogar em casa quando esta se muda de uma cidade para outra, uma situação comum em locais onde os times são franquias particulares. Lacerda et al. (2008) abordam a existência do Home Advantage nos Jogos Pan Americanos e seus efeitos residuais nos jogos seguintes.

Os artigos científicos que tratam do Home Advantage têm sido estruturados basicamente a partir do levantamento de hipóteses que expliquem as razões de as equipes mandantes terem desempenho superior às visitantes, na maioria das vezes em que competem. Várias hipóteses diferentes são levantadas. As principais hipóteses destes trabalhos têm sido:

- As equipes mandantes levam vantagem por conhecerem melhor a característica do local de competição, como condições do campo de jogo, do traçado da pista, etc.

- Os julgamentos dos árbitros das competições são mais favoráveis para as equipes e atletas mandantes (Dawson et al., 2007). Esta hipótese é particularmente importante em modalidades esportivas que avaliam os competidores através de notas, envolvendo mais fortemente o julgamento subjetivo, como ginástica e saltos ornamentais, por exemplo. Embora as federações internacionais destes esportes tenham códigos rígidos de pontuação, com o objetivo de retirar do árbitro a responsabilidade sobre a medição de desempenho e torná-lo apenas um observador da execução ou não de um conjunto de movimentos prédeterminados, o levantamento dos dados relativos a estas competições mostra que esta hipótese é relevante para a verificação de Home Advantage.

- O comportamento dos torcedores influencia de forma positiva o desempenho das equipes mandantes, devido à maior motivação dos atletas destas equipes (Nevill et al., 2002). Por tratar-se de uma hipótese que se fundamenta em um aspecto comportamental, sua verificação através de ferramentas quantitativas é mais difícil de ser realizada. Neste caso, a maior dificuldade está em correlacionar os dados de Home Advantage com o nível de incentivo do público que assiste à competição esportiva. 
Nestes estudos, as hipóteses elaboradas nem sempre são comprovadas através dos testes de hipótese estatística, ou seja, há outros fatores determinantes de Home Advantage que não têm sido considerados nestes artigos científicos. Além disso, em diferentes artigos que tratam do tema, as análises apresentadas tomam os dados com base em metodologias que interpretam a sua obtenção de forma estática, isto é, não são observadas tendências entre os dados obtidos. Não se observa, por exemplo, uma possível evolução do desempenho esportivo de determinada nação, ou, ainda, se o seu desempenho é influenciado por alguma externalidade em determinado período.

O efeito residual de uma preparação bem realizada em edições posteriores dos jogos, assim como o caso dos atletas profissionais naturalizados, são fatores que não vêm sendo estudados pelos artigos científicos. O desempenho dominicano nos jogos do Rio de Janeiro em 2007 foi significativamente superior aos dos anos anteriores a 2003. Estes efeitos serão comentados na análise de dados e conclusões.

Sobre a classificação das equipes participantes de competições esportivas de grande porte, tem-se que a utilização de um ranking lexicográfico para classificar as nações participantes dos jogos é utilizada pelos comitês organizadores e consagrada pelo público e mídia como a ordenação oficial, tanto para os Jogos Pan Americanos como para os Jogos Olímpicos. Alguns autores já propuseram alternativas para esta classificação. Lozano et al. (2002) utilizaram a metodologia DEA para elaborar um ranking dos Jogos Olímpicos, assim como o fez Lins et al. (2003). Churilov \& Flitman (2006) utilizaram diferentes critérios de classificação e desempate entre os competidores. Trabalhos mais recentes aplicaram modelos DEA com input unitário e avaliação cruzada (Soares de Mello et al., 2008) e ainda modelos DEA com input unitário e avaliação cruzada com separação de modalidades (Soares de Mello et al., 2009).

Sobre a atribuição de pesos para as medalhas, com o objetivo de montar-se um ranking baseado em pontuação, Balmer et al. (2001) utilizou-se de ponderação simples, dando às medalhas de ouro mais importância que às de prata, e a estas mais que às de bronze, em intervalos iguais (Lacerda et al., 2008).

\section{METODOLOGIAS DE ORDENAÇÃO}

Conforme apontado na Seção 2, o método classificatório atualmente utilizado nos Jogos Pan Americanos, tal como nos Jogos Olímpicos, é baseado no método lexicográfico. Este método apresenta duas características fundamentais: assume que o decisor é apto a ordenar cada critério segundo sua importância observada; e estabelece um ranking de acordo com o critério considerado como o mais importante, independentemente dos outros critérios. Em caso de empate, parte-se para o segundo critério mais importante e assim por diante. No caso dos jogos Pan Americanos, assim como nas olimpíadas, o critério mais importante é o número de medalhas de ouro. $\mathrm{O}$ segundo mais importante refere-se ao número de medalhas de prata e o terceiro representa as medalhas de bronze. Isso implica numa valorização excessiva das medalhas de ouro que gera distorções na ordenação final dos países na competição. 
Esta ordenação, que é a referencial para o estudo do Home Advantage, pode não ser suficiente para uma análise mais completa. Um país pode estar gradualmente melhorando sua performance nos Jogos e não ter sua classificação acompanhando este desenvolvimento. Isto porque se um país obtiver uma única medalha de ouro, terá um desempenho considerado superior a de outro com 10 de prata e 20 de bronze, por exemplo.

No entanto, conforme descrito em Soares de Mello et al. (2008), uma valorização maior da medalha de ouro deve ser considerada, por ser a vitória o objetivo final dos competidores. Todavia, com o intuito de minimizar a supervalorização da medalha de ouro em relação às demais, considera-se que a diferença entre os pesos da medalha de ouro em relação à medalha de prata e desta em relação à de bronze devem ser diferentes.

Métodos multicritério podem, portanto, sofrer adaptações e serem utilizados para tentar contornar o inconveniente do método lexicográfico. Kao \& Hung (2007) fazem uso de um método híbrido que considera na formação dos pesos tanto os aspectos subjetivos, quanto os objetivos. Gomes et al. (2008) também utilizam este princípio para criar um índice multicritério de bem estar social rural.

Outras propostas encontradas na literatura usam metodologia DEA e visam contornar tais distorções e criar um sistema de avaliação mais "justo". Recentemente, Churilov \& Flitman (2006) agregaram ao modelo, além das relações entre as medalhas, o PIB dos países e tamanho da população.

Chaves et al. (2008) apresentaram uma comparação entre três diferentes metodologias de ordenação, com objetivo de estabelecer o método mais apropriado para análise de Home Advantage. Foram aplicadas as metodologias DEA, um método multicritério derivado do VIP Analysis (Variable Interdependent Parameter) e um método ingênuo, que é um método de Borda invertido, onde os pesos de cada critério são definidos pelo decisor e a pontuação final de cada alternativa é dada pela soma ponderada dos critérios.

O primeiro método analisado em Chaves et al. (2008) é um aprimoramento do método ingênuo. O método ingênuo consiste em atribuir peso 3 para medalhas de ouro, 2 para a de prata e 1 para a de bronze. A variação deste método, denominado método de pesos fixos com diferença de valor, pretende realizar uma valorização maior da medalha de ouro, por se entender a vitória como o objetivo final dos competidores (Soares de Mello et al., 2008). Desta forma, a diferença entre os pesos da medalha de ouro em relação à medalha de prata e desta em relação à de bronze devem ser diferentes. Por isso, foram utilizados os pesos 4, 2 e 1 para as medalhas de ouro, prata e bronze, respectivamente. A pontuação final de cada alternativa é dada pela soma ponderada do número de medalhas. Em caso de empate, continua-se utilizando o método lexicográfico nos mesmos moldes que é normalmente utilizado.

Em seguida, foi avaliado o uso da metodologia DEA, baseado em programação matemática, cujo objetivo é medir a eficiência de um conjunto de unidades produtivas (unidades de tomada de decisão), denominadas de DMUs (do inglês “Decision Making Units"), que consomem múltiplos inputs (insumos, recursos) para produzir múltiplos outputs (produtos). Ou seja, é um modelo para 
a análise da eficiência (Charnes et al., 1978). O modelo DEA utilizado e justificado em Chaves et al. (2008) é um modelo orientado a inputs.

Deve-se lembrar que o valor máximo de eficiência encontrado para uma dada DMU é 1 (esta DMU é considerada eficiente), não sendo permitido que a eficiência de nenhuma outra DMU considerada seja superior à da DMU eficiente (exceção feita ao modelo de supereficiência, Zhu (2001)). Esta característica não é interessante em uma análise de Home Advantage, pois ao longo dos anos cada país tem sempre sua performances máxima limitada a 1, principalmente no caso de países como os Estados Unidos, que historicamente lideram os rankings de medalhas em jogos esportivos, sediarem as competições.

Assim, a metodologia DEA não se mostrou adequada ao objetivo pretendido. Finalmente, Chaves et al. (2008) apresentam o método multicritério derivado do VIP Analysis, que permite uma atribuição para os pesos dos critérios, tão benevolente quanto possível, para a alternativa em análise. Isto porque a análise é individual e não comparativa. Desta forma, é possível que a atribuição ótima dos pesos para uma alternativa seja ainda melhor para uma outra alternativa do modelo. Além disso, não possui uma restrição de valor máximo de eficiência. Por considerar ser esta uma característica que se enquadra perfeitamente no estudo deste trabalho, esta foi a abordagem adotada.

A formulação geral do modelo multicritério utilizado no VIP Analysis é apresentada no modelo de programação linear (1):

$$
\operatorname{Max} Z=V(a i, k)=\sum_{j=1}^{n} k_{j} v_{j}\left(a_{i}\right)
$$

s.a.

onde:

$$
\begin{aligned}
& \sum_{j=1}^{n}=1 \\
& k_{j} \geq 0, j=1, \ldots, n
\end{aligned}
$$

$a_{1}, \ldots, a_{m}$ são as alternativas;

$v(\cdot)$ é uma função valor com único argumento de cada alternativa associada ao $j$-ésimo critério e assinala um valor numérico a cada performance;

$k_{j}$ é o parâmetro de importância (peso) do $j$-ésimo critério;

$n$ é o total de critérios.

Neste modelo, espera-se que o agente de decisão (AD) possa estabelecer, pelo menos uma ordenação dos critérios, agregando-se ao modelo os valores subjetivos do AD. Caso contrário, a solução do modelo é trivial, dada pela atribuição de peso 1 ao critério no qual a variável tem melhor desempenho. Este modelo sofreu algumas adaptações de modo a espelhar a proposta do trabalho de Chaves et al. (2008) de gerar uma agregação para os resultados das competições dos Jogos Pan Americanos, capaz de valorizar ao máximo os resultados obtidos por cada país 
e de, ao mesmo tempo, reflitir a noção de importância tradicionalmente associada ao valor das medalhas (Soares de Mello et al., 2008). O conjunto de modelos de Programação Linear (PL) apresentados em 3.1 refletem tais premissas.

\subsection{Método Multicritério derivado do VIP Analysis}

Para obter-se uma ordenação para os resultados das competições dos Jogos Pan-americanos, construiu-se um novo modelo de Programação Linear (PL), visando diminuir as possíveis situações injustas que ocorrem em consequência da utilização do modelo lexicográfico, além de fornecer aos países um tipo de informação que considere a importância do desempenho global das delegações. O modelo (2) é denominado método multicritério derivado do VIP Analysis, e incorpora tanto aspectos subjetivos, como objetivos para a determinação dos pesos dos critérios.

$$
\operatorname{Max} Z=V(a i, k)=\sum_{j=1}^{3} k_{j} v_{j}\left(a_{i}\right)
$$

s.a.

$$
\begin{aligned}
& k_{1} \geq k_{2} \\
& k_{2} \geq k_{3} \\
& k_{1}-k_{2} \geq k_{2}-k_{3} \\
& \sum_{j=1}^{3} k_{j}=1 \\
& k_{j} \geq 0, j=1, \ldots, 3
\end{aligned}
$$

onde:

$a_{1}, \ldots, a_{m}$ são os países que ganharam medalhas nos Jogos Pan Americanos em estudo;

$k_{j}$ é o parâmetro de importância (peso) do $j$-ésimo critério ( $j=1$ (ouro), 2 (prata), 3 (bronze)); $v_{j}\left(a_{i}\right)$ é obtido pela normalização do número de medalhas do tipo $j$ ganhas pelo país $a_{i}$ e o total geral de medalhas $j$.

A solução do modelo (2) é obtida pelo VIP Analysis. Para tal, basta que o conjunto de alternativas considerado seja o conjunto de países, os critérios sejam o número total de medalhas de ouro, prata e bronze conquistado. Desta forma, o modelo (2) é uma variação do modelo (1), que considera que o tomador de decisão se sente confortável para fornecer uma ordenação dos critérios. Uma discussão completa sobre os resultados do VIP Analysis pode ser encontrada em Dias \& Clímaco (2000). Campos \& Almeida (2006) e Chaves et al. (2010) descrevem estudos que elucidam a natureza das conclusões obtidas pela metodologia.

A primeira e a segunda restrição do modelo representam o fato usualmente aceito de que as medalhas não são igualmente importantes. A medalha de ouro é mais importante que a de prata e essa é, por sua vez, mais importante que a de bronze. Além disso, a importância relativa delas não é a mesma, pois a vitória é assumida como o principal objetivo dos competidores. Assim, 
a terceira restrição garante que a diferença de importância entre as medalhas de ouro e prata não seja menor que a diferença entre prata e bronze. É possível demonstrar a existência de redundância entre a segunda e a terceira restrição. Porém, por motivo de clareza, as duas serão mantidas no modelo.

Compete destacar que este uso do VIP Analysis é uma redução do escopo da metodologia e foge ao seu propósito original. Contudo, seu uso é benéfico para a resolução dos modelos necessários para a geração dos resultados pretendidos. Os dados referentes ao desempenho de cada país foram normalizados, de forma a permitir sua utilização.

Nesta análise, dos resultados fornecidos pelo método, o interesse principal é a coluna chamada Max Value, denominada índice de desempenho.

O modelo (2) propicia uma análise individual de cada alternativa em estudo, permitindo uma atribuição para os pesos dos critérios tão benevolente quanto possível. Desta forma, é possível uma atribuição para os pesos para uma alternativa que permita que uma outra alternativa seja ainda mais favorecida. O resultado otimizante de cada alternativa só depende da performance da alternativa em análise. Os resultados encontrados pelo método para a Republica Dominicana, por exemplo, estão apresentados na Tabela 1. De um modo geral, os pesos atribuídos pelo modelo a cada alternativa, em cada uma das edições dos Jogos Pan Americanos, não sofreram grandes variações, motivo pelo qual não foram apresentados neste trabalho. Para a República Dominicana, por exemplo, os pesos atribuídos foram iguais para os três tipos de medalha em todas as edições, desde 1975, como mostrado na Figura 1.

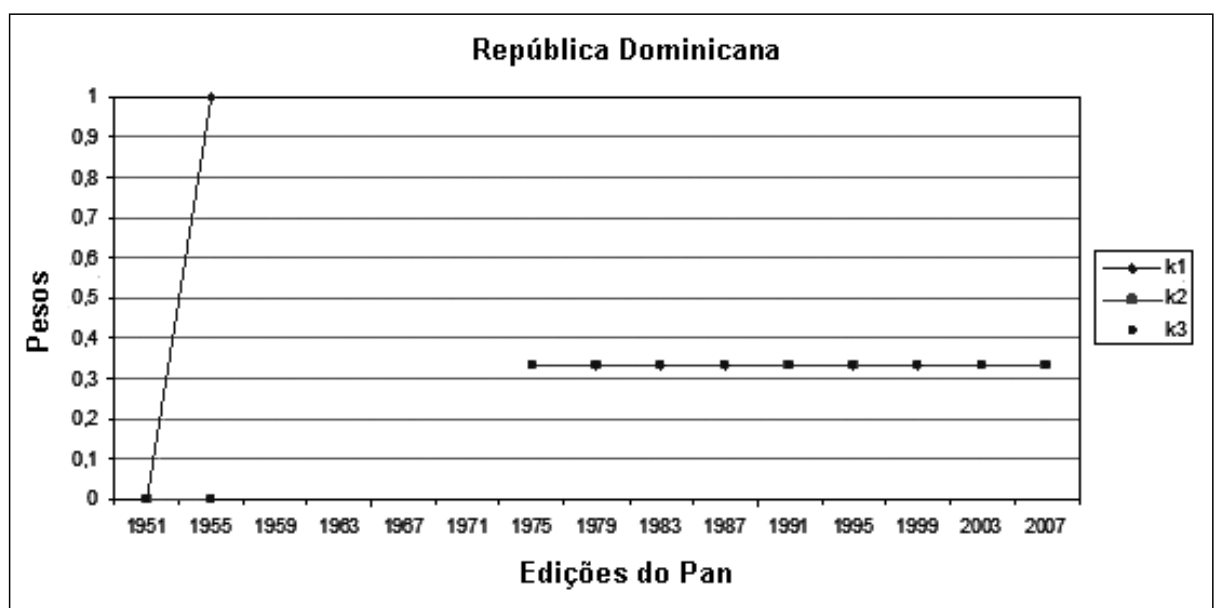

Figura 1 - Evolução dos pesos atribuídos à República Dominicana pelo método derivado do VIP Analysis.

\section{RESULTADOS OBTIDOS}

A Tabela 1 mostra a pontuação obtida pela República Dominicana segundo o método derivado VIP Analysis em todas as edições dos jogos Pan Americanos de que participou. Nos anos de 1951, 1963 e 1967 o país não participou dos Jogos. 
Tabela 1 - Índice de Desempenho para a República Dominicana.

\begin{tabular}{|c|c|c|c|c|c|}
\hline \multirow{2}{*}{ Ano } & \multirow{2}{*}{ País sede } & \multicolumn{3}{|c|}{ Total de medalhas } & \multirow{2}{*}{$\begin{array}{c}\text { Índice de } \\
\text { desempenho }\end{array}$} \\
\hline & & Ouro & Prata & Bronze & \\
\hline 1951 & Argentina & & & & - \\
\hline 1955 & México & 1 & 0 & 1 & 0,007 \\
\hline 1959 & Estados Unidos & 0 & 0 & 0 & 0 \\
\hline 1963 & Brasil & & & & - \\
\hline 1967 & Canadá & & & & - \\
\hline 1971 & Colômbia & 0 & 0 & 0 & 0 \\
\hline 1975 & México & 0 & 1 & 7 & 0,012 \\
\hline 1979 & Porto Rico & 0 & 5 & 10 & 0,019 \\
\hline 1983 & Venezuela & 0 & 7 & 7 & 0,016 \\
\hline 1987 & Estados Unidos & 0 & 3 & 9 & 0,011 \\
\hline 1991 & Cuba & 0 & 5 & 4 & 0,002 \\
\hline 1995 & Argentina & 1 & 1 & 5 & 0,005 \\
\hline 1999 & Canadá & 1 & 3 & 6 & 0,009 \\
\hline 2003 & República Dominicana & 10 & 12 & 19 & 0,0037 \\
\hline 2007 & Brasil & 6 & 6 & 17 & 0,0018 \\
\hline
\end{tabular}

A Figura 2 auxilia a visualização dos pontos listados na Tabela 1. Foram acrescentadas duas linhas de tendência. Uma delas é uma reta de regressão linear simples. A outra é uma linha de médias móveis com índice 2.

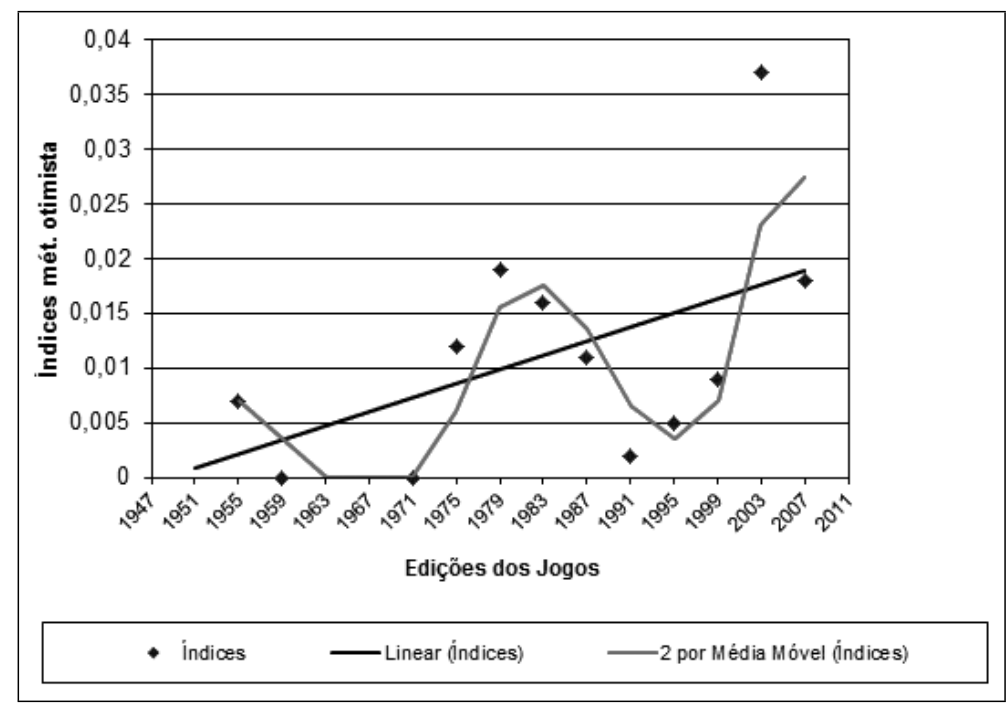

Figura 2 - Evolução do desempenho da República Dominicana pelo método derivado do VIP Analysis.

Além da República Dominicana, foram testados os dados de desempenho de outro país nos jogos Pan Americanos - a Argentina, escolhida por ter um maior número de participações nesses 
jogos e por tê-los sediado em duas oportunidades, 1951 e 1995. A Tabela 2 revela os índices de desempenho para a Argentina.

A Figura 3 ilustra os dados apresentados na Tabela 2.

Tabela 2 - Índice de Desempenho para a Argentina.

\begin{tabular}{|c|c|c|c|c|c|}
\hline \multirow{2}{*}{ Ano } & \multirow{2}{*}{ País sede } & \multicolumn{3}{|c|}{ Total de medalhas } & \multirow{2}{*}{$\begin{array}{l}\text { Índice de } \\
\text { desempenho }\end{array}$} \\
\hline & & Ouro & Prata & Bronze & \\
\hline 1951 & Argentina & 68 & 44 & 38 & 0,472 \\
\hline 1955 & México & 27 & 31 & 15 & 0,197 \\
\hline 1959 & Estados Unidos & 9 & 19 & 11 & 0,084 \\
\hline 1963 & Brasil & 8 & 15 & 16 & 0,112 \\
\hline 1967 & Canadá & 8 & 14 & 12 & 0,06 \\
\hline 1971 & Colômbia & 6 & 4 & 12 & 0,036 \\
\hline 1975 & México & 3 & 5 & 7 & 0,023 \\
\hline 1979 & Porto Rico & 12 & 7 & 17 & 0,05 \\
\hline 1983 & Venezuela & 2 & 11 & 22 & 0,024 \\
\hline 1987 & Estados Unidos & 12 & 14 & 22 & 0,047 \\
\hline 1991 & Cuba & 11 & 15 & 29 & 0,066 \\
\hline 1995 & Argentina & 40 & 45 & 74 & 0,114 \\
\hline 1999 & Canadá & 25 & 19 & 28 & 0,076 \\
\hline 2003 & República Dominicana & 16 & 20 & 27 & 0,058 \\
\hline 2007 & Brasil & 11 & 15 & 33 & 0,033 \\
\hline
\end{tabular}

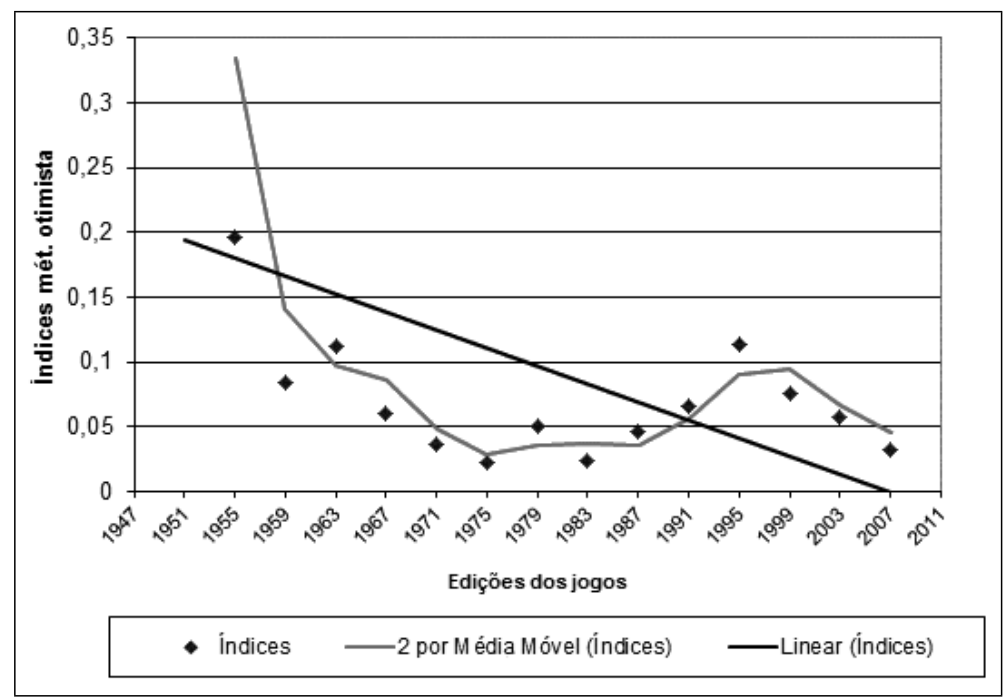

Figura 3 - Evolução do desempenho da Argentina pelo método derivado do VIP Analysis.

No caso da República Dominicana (que sediou os jogos em 2003), não é possível observar facilmente através da Tabela 1 e da Figura 2 se houve melhora em seu desempenho naquela oportunidade. A reta de regressão pelos mínimos quadrados indica uma tendência de evolução no 
desempenho do país ao longo dos anos sem, no entanto, indicar que o fato de sediar os jogos em 2003 tenha influenciado no desempenho do país. Além disso, a técnica das médias móveis não indicou o mesmo fato, e sim uma oscilação entre períodos de bom desempenho alternado com outros piores. Ou seja, embora nas edições próximas ao ano de 2003 a delegação Dominicana tenha tido um bom desempenho nas competições, não é possível diferenciar se tal resultado é fruto de Home Advantage, ou se não passa de mais uma melhoria cíclica de desempenho, como ocorreu nas edições de 1979 a 1983 . Vale lembrar que neste período o país foi sempre visitante nas competições, uma vez que os jogos de 1979 foram disputados em Porto Rico e os de 1983 foram disputados na Venezuela.

Observa-se, no entanto, a ocorrência de Home Advantage nos indicadores de desempenho da Argentina. Na primeira edição dos jogos (Buenos Aires, 1951), o desempenho argentino teve seu maior índice, e o efeito residual deste elevado padrão foi observado ainda nas duas edições seguintes. A partir daí, no período de 1967 até 1991, o padrão apresentado manteve-se quase constante, com desempenhos similares entre si. Este desempenho voltou a um patamar elevado em 1995, quando os jogos foram disputados em Mar del Plata. Nas edições seguintes, em 1999, 2003 e 2007, o desempenho começou a retornar para os padrões apresentados até 1991, indicando a influência do efeito Home Advantage no ano de 1995.

Ainda com base na Figura 3 e nas linhas de tendência, observa-se que a regressão pelos mínimos quadrados leva a dados irreais a partir de 2007, com previsão até de medalhas negativas, numa influência certa de um ponto muito acima dos outros (1951). Já o modelo de médias móveis teve resultados mais satisfatórios, refletindo as variações de desempenho da delegação ao longo dos anos. Como se trata de uma delegação com maior número de participações nos jogos que a República Dominicana, o uso da metodologia derivada do VIP Analysis foi eficiente para encontrar o efeito Home Advantage.

\section{CONCLUSÕES}

A análise através de uma ferramenta que permite visualizar as tendências denota que a preparação mais elaborada do país sede de uma competição de grande porte também é um fator que contribui para acentuar a vantagem de se jogar em casa. Além disso, frequentemente os países-sede de grandes eventos que não contam com investimentos maciços na prática esportiva recorrem a estratégias que escapam do foco dos artigos publicados sobre o tema.

O caso que ilustra de forma mais clara esta situação é a estratégia da República Dominicana de naturalização de atletas de ponta para defender sua bandeira, mediante compensação financeira. O país naturalizou alguns atletas chineses de tênis de mesa para defender sua bandeira nos jogos de Santo Domingo, em 2003. Com isso, ganhou medalhas em uma modalidade em que não contava com nenhum cidadão nato figurando na elite deste esporte, não só nos jogos de 2003, como também no Rio de Janeiro em 2007. Atento a estas movimentações de atletas, que ameaçam a integridade de competições entre nações, os Comitês Olímpicos nacionais e o Comitê Olímpico Internacional estudam medidas restritivas à participação de atletas naturalizados em suas competições, estabelecendo um tempo mínimo de vivência do atleta em seu novo país, 
ou até a exigência de existência de algum vínculo familiar do atleta com sua nova pátria. Um fator complicador da imposição destas medidas é a legislação de cada país para naturalizar os seus cidadãos, matéria que os comitês não têm competência legal para tratar.

Como estudo futuro, propõe-se trabalhar com o método multicritério derivado do VIP Analysis para situações com um volume de dados disponível maior, como, por exemplo, os Jogos Olímpicos de Verão. Pretende-se também utilizar o método multicritério derivado do VIP Analysis como fonte de dados para sistemas de previsão de desempenho, baseados no aprendizado de redes neurais.

\section{REFERÊNCIAS}

[1] Balmer NJ, Nevill AM \& Williams AM. 2001. Home advantage in the Winter Olympics (1908-1998). Journal of Sports Sciences, 19(2): 129-139.

[2] Balmer NJ, Nevill AM \& Williams AM. 2003. Modeling home advantage in the Summer Olympic Games. Journal of Sports Sciences, 21(6): 469-478.

[3] Campos V \& Almeida AT. 2006. Modelo Multicritério para localização de Nova Jaguariba com VIP Analysis. Pesquisa Operacional, 16: 91-107.

[4] Charnes A, Cooper WW \& Rhodes E. 1978. Measuring the Efficiency of Decision-Making Units. European Journal of Operational Research, 2: 429-444.

[5] Chaves MCCC, Lacerda FG, Gomes Junior S, Soares de Mello JCC \& Sant' Anna AP. 2008. Uso de método baseado em DEA e multicritério para avaliação de resultados em competições esportivas: o caso dos jogos Pan-Americanos de 2007 in: XL Simpósio Brasileiro de Pesquisa Operacional. SPBO 2008. João Pessoa.

[6] Chaves mCC, Ramos TG, Barros TD \& Soares de Mello JCCB. 2010. Uso integrado de dois métodos de apoio à decisão multicritério: VIP Analysis e MACBETH. Pesquisa Operacional para o Desenvolvimento, 2(2): 89-99.

[7] Churilov L \& Flitman A. 2006. Towards fair ranking of Olympics achievements: The case of Sydney 2000. Computers and Operations Research, 33(7): 2057-2082.

[8] DaWson PA, Dobson SB, GodDARD JC \& WILSON JD. 2007. Are football referees really biased and inconsistent? Evidence on the incidence of disciplinary sanction in the English Premier League. Journal of the Royal Statistical Society. Series A: Statistics in Society, 170(1): 231-250.

[9] DiAS LC \& CLÍMACo JN. 2000. Additive Aggregation with variable Interdependent Parameteres: the VIP Analysis Software. Journal of Operational Research Society, 51(9): 1070-1082.

[10] Gomes EG, SoAres de Mello JCCB \& Mangabeira JAC. 2008. Índice multicritério de bem estar social rural em um município da região amazônica. Pesquisa Operacional, 28(1): 141-160.

[11] KAO C \& Hung H-T. 2007. Management performance: An empirical study of the the manufacturing companies in Taiwan. Omega, 35: 152-160.

[12] Lacerda FG, Chaves MCC, Gomes Junior SF \& Soares de Mello JCCB. 2008. Aplicação de método multicritério otimista para avaliação de desempenho e ocorrência de home advantage em esportes: o caso dos jogos Pan-Americanos. Anais do XXVIII Encontro Nacional de Engenharia de Produção. 
[13] Li Y, LiAng L, CHEN Y \& MORITA H. 2008. Models for measuring and benchmarking olympics achievements. Omega, 36(6): 933-940.

[14] Lins MPe, Gomes EG, Soares de Mello JCCB \& Soares de Mello AJR. 2003. Olympic ranking based on a zero sum gains DEA model. European Journal of Operational Research, 148(2): 312-322.

[15] LOVELL CAK \& PASTOR JT. 1999. Radial DEA models without inputs or outputs. European Journal of Operational Research, 118: 46-51.

[16] Lozano S, Villa G, Guerrero F \& Cortés P. 2002. Measuring the performance of nations at the Summer Olympics using data envelopment analysis. Journal of the Operational Research Society, 53(5): 501-511.

[17] Nevill AM, Balmer NJ \& Williams M. 2002. The influence of crowd noise and experience upon refereeing decisions in football. Psychology of Sport and Exercise, 3: 261-272.

[18] Pollard R. 2002. Evidence of a reduced home advantage when a team moves to a new stadium. Journal of Sports Sciences, 20(12): 969-973.

[19] Soares de Mello JCCB, Gomes EG, Angulo Meza L \& Biondi Neto L. 2008. Cross evaluation using weight restriction in unitary input DEA models: Theoretical aspects and application to Olympic Games ranking. WSEAS Transactions on Systems, 7(1): 31-39.

[20] Soares de Mello JCCB, Angulo Meza L \& Branco Da Silva BP. 2009. A ranking for the Olympic Games with unitary input DEA models. IMA Journal of Management Mathematics, 20(2): 201-211.

[21] ZHU J. 2001. Super-efficiency and DEA sensitivity analysis. European Journal of Operational Research, 129: 443-455. 